\title{
Management of Asymptomatic HIV Infection
}

\author{
Michael S. Simberkoff \\ Infectious Diseases Section and Research Center for AIDS and HIV Infection, New York \\ Department of Veterans Affairs Medical Center, and the Department of Medicine, \\ NYU School of Medicine, New York, New York, USA
}

\section{Contents}

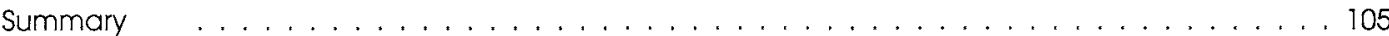

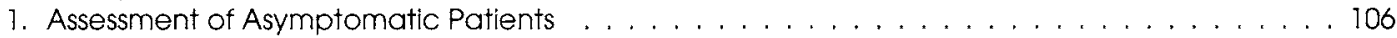

2. Antiretroviral Treatment . . . . . . . . . . . . . . . . . . . . . . . 108

2.1 Monotherapy with Antiretroviral Drugs . . . . . . . . . . . . . . . . . . . . . 108

2.2 Combination Therapy with Antiretroviral Drugs . . . . . . . . . . . . . . . . . . . . . . . 109

3. Additional Treatment and Prophylaxis . . . . . . . . . . . . . . . . . . . 112

3.1 Tuberculosis . . . . . . . . . . . . . . . . . . . . . . . 112

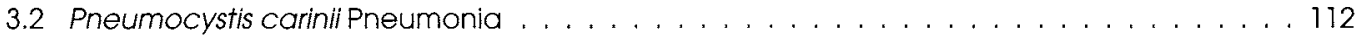

3.3 Toxoplasma Encephalitis . . . . . . . . . . . . . . . . . . . . 113

3.4 Disseminated Mycobacterium avium Complex Disease . . . . . . . . . . . . . . . . . 113

3.5 Cytomegalovirus Disease . . . . . . . . . . . . . . . . . . . . . . 113

3.6 Invasive Fungal Infections . . . . . . . . . . . . . . . . . . . . . . . . 113

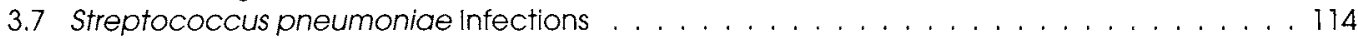

4. Conclusions . . . . . . . . . . . . . . . . . . . . . . . . . 114

\section{Summary}

Patients are asymptomatic during the majority of HIV infection. The virus, however, is actively replicating, producing over 1 billion particles per day during this steady-state of the disease. Management of asymptomatic HIV infection involves initial recognition of infection and counselling to prevent its further spread.

When HIV infection has been confirmed, assessment consists of a careful history, physical examination and laboratory studies. The latter include serological tests for past exposure to infectious agents such as syphilis, hepatitis viruses, cytomegalovirus and toxoplasmosis. A purified protein derivative (PPD) skin test should also be performed. The immunological and virological status of the patient is assessed by quantifying CD4+ count and plasma HIV RNA levels. With the aid of these data, a decision can be made on the use of antiretroviral therapy.

Didanosine (ddI) is the only currently acceptable monotherapy. Combinations of antiretroviral drugs which have undergone evaluation include zidovudine (AZT) plus didanosine, zidovudine plus zalcitabine (ddC), zidovudine plus lamivudine (3TC), didanosine plus lamivudine, zidovudine plus didanosine plus nevirapine, zidovudine plus saquinavir, zidovudine plus zalcitabine plus saquinavir 
(D4T), zidovudine plus ritonavir, zidovudine plus lamivudine plus indinavir, and ritonavir plus saquinavir.

The optimum combination treatment has not been defined, but many appear promising. In addition, treatment for subclinical infections and prophylaxis should be administered. These include treatment of all PPD-positive patients with isoniazid $300 \mathrm{mg} /$ day orally for 1 year; prophylaxis against Pneumocystis carinii pneumonia for all patients with CD4+ counts $<200 / \mathrm{mm}^{3}$ with cotrimoxazole (trimethoprim plus sulfamethoxazole) one double-strength tablet daily, dapsone or dapsone plus trimethoprim; prophylaxis against toxoplasmosis in patients with CD4+ counts $<100 / \mathrm{mm}^{3}$ with cotrimoxazole or with dapsone plus pyrimethamine; prophylaxis against disseminated Mycobacterium avium complex disease in patients with CD4+ counts $<75 / \mathrm{mm}^{3}$ with azithromycin $1200 \mathrm{mg}$ orally once weekly, clarithromycin $500 \mathrm{mg}$ orally twice daily, or rifabutin $300 \mathrm{mg} /$ day orally; and prophylaxis against Streptococcus pneumoniae pneumonia and bacteraemia by administration of the 23-valent pneumococcal vaccine.

Although cytomegalovirus disease occurs in most patients with advanced HIV disease $\left(\mathrm{CD} 4+<50 / \mathrm{mm}^{3}\right)$, primary prophylaxis is not recommended because of conflicting data concerning the efficacy of oral ganciclovir. Primary prophylaxis against cryptococcosis is not recommended because of the low incidence of this infection.

More than 15 years have elapsed since the first cases of AIDS were described in Los Angeles and New York. HIV, the cause of AIDS, was discovered in laboratories in France and the US in 1983. By 1996 it was estimated that 24 million individuals had already been infected by HIV throughout the world, 20 million infected individuals were living, and 10000 new infections were occurring each day. ${ }^{[1]}$ The vast majority of these individuals, if untreated, will experience inexorable virological, immunological and clinical deterioration culminating in an opportunistic infection, malignancy, wasting syndrome, dementia and death.

Patients can experience transient symptoms soon after acquisition of primary HIV infection. They generally develop symptoms during advanced diseases when they develop minor or major opportunistic infections, malignancy, wasting syndrome, encephalopathy or myelopathy. They are, however, asymptomatic for the majority of the time that HIV infection is present. It was thought that HIV was latent during most of this asymptomatic period. It is now recognised, however, that the virus is actively replicating, producing over a billion particles per day and destroying CD4+ cells at a prodigious rate during this steady-state of the disease. ${ }^{[2,3]}$

\section{Assessment of Asymptomatic Patients}

Management of patients during the asymptomatic period involves:

- recognition of the infection

- counselling to prevent its further spread

- diagnosis and treatment of coexistent disease

- assessment of the virological and immunological stage of HIV infection

- initiation of appropriate antiretroviral drugs

- initiation of appropriate prophylaxis to prevent infections.

For the most part, HIV testing in industrialised countries, such as the US, is voluntary. However, involuntary testing takes place on all blood donated for transfusion, in the military, in some prisons, among immigrants applying for permanent residence status and for insurance purposes. In federal Veterans Affairs medical facilities and in most states, written informed consent is required before voluntary HIV testing can be performed. Testing is advised for individuals who engage in high risk activities such as unprotected homosexual or pro- 
miscuous heterosexual contact, and intravenous drug use. It is also recommended for patients with other sexually transmitted diseases and for all pregnant women.

HIV counselling is done in association with testing (table I). Counselling is done both before and after the test results are known. This 2-stage counselling is thought to be useful to give, and to reinforce, information about the limitations of the test, the nature of HIV infection and its relation to AIDS, the routes by which HIV infection is spread and the means to prevent this.

Once HIV infection is detected, the patient must be assured that they will have appropriate followup and treatment. Initial evaluation includes a thorough history and physical examination, with emphasis on symptoms and signs suggestive of the infections and malignancies that occur with increased frequency in HIV-infected patients. In addition, the clinician evaluates the patient's prior exposure to infectious diseases [by serological tests for syphilis, hepatitis B virus, hepatitis $\mathrm{C}$ virus, cytomegalovirus (CMV) and toxoplasmosis, and by a tuberculin skin test] and initiates appropriate treatment. Finally, the immunological status and HIV viral load of the patients are determined.

The immunological status of an HIV-infected patient is assessed by means of the CD4+ count, whose significance has been validated in several studies. For example, Fahey et al. ${ }^{[4]}$ reported that the CD4+ count was the best of several cellular and serological predictors of clinical progression in the Multicenter AIDS Cohort Study (MACS), a large cohort of homosexual individuals who were recruited in 4 US cities between 1984 and 1985 and followed since. In addition, CD4+ count is inversely related to the risk of several opportunistic infections, including Pneumocystis carinii pneumonia (PCP), toxoplasmosis, cryptococcosis, disseminated Mycobacterium avium complex (DMAC) and CMV infection. ${ }^{[5]}$ Monitoring for and prophylaxis against these are initiated as the CD4+ count falls.

Plasma HIV RNA levels provide important prognostic information. Three assay systems have been
Table I. Assessment of asymptomatic patients

Counsel and test for HIV antibody

Careful history and physical examination

Serological tests for syphilis, hepatitis viruses, cytomegalovirus and toxoplamosis

Skin test (PPD) for tuberculosis

CD4+ count

Quantify plasma HIV RNA

Abbreviation: $\mathrm{PPD}=$ purified protein derivative.

developed to quantify plasma HIV RNA. These are a branched DNA (bDNA) assay (Chiron), a reverse transcriptase polymerase chain reaction assay (Roche), and a nucleic acid sequence-based amplification assay (Organon Teknika). A recent review describes, compares, and makes recommendations for appropriate use of these tests to quantify HIV RNA. ${ }^{[6]}$ Plasma HIV RNA levels are generally measured 3 to 4 times per year in association with the CD4+ count. They may also be quantified 4 to 6 weeks after initiation of a new or altered antiretroviral regimen to assess the response to therapy (see below).

In 1995, Mellors et al., ${ }^{[7]}$ using a bDNA assay, showed that the levels of HIV RNA in plasma obtained soon after a known date of seroconversion predicted the outcome of 62 patients. More recently, this group extended the studies of plasma HIV RNA levels to a large number of the MACS patients. ${ }^{[8]}$ They demonstrated a direct relationship between the levels in plasma of HIV RNA in these patients and their risk for progression to AIDS or death. Further, they found that plasma HIV RNA levels were independent of CD4+ counts and were better predictors of progression than the latter.

Similar findings were reported from a multicentre haemophilia cohort study where a reverse transcriptase polymerase chain reaction assay system was used to quantify HIV RNA. In this study, O'Brien et al. ${ }^{[9]}$ found that the risk of progression to AIDS among the patients with HIV-infected haemophilia was related to HIV RNA and to age but was not related to CD4+ count.

HIV RNA measurements were also assessed in retrospective studies of patients enrolled in AIDS 
Clinical Trials Group (ACTG) protocol 116A, a randomised trial of zidovudine (AZT) compared with didanosine (ddI) in patients with little or no prior zidovudine experience. ${ }^{[10]}$ HIV RNA was measured by both bDNA and reverse transcriptase polymerase chain reaction in this study, and the results obtained by these 2 methods were strongly correlated. Baseline HIV RNA and CD4+ counts proved to be independent predictors of disease progression in this study.

Changes in plasma HIV RNA levels and CD4+ counts predict clinical outcomes during treatment with antiretroviral drugs. This was demonstrated in a retrospective analysis of the Department of Veterans Affairs (VA) Cooperative Studies Program trial that compared early with delayed zidovudine treatment. ${ }^{[11]}$ More recently, the value of HIV RNA levels for predicting prognosis and treatment effect was confirmed in ACTG protocol 175, a trial comparing monotherapy versus combinations of antiretroviral drugs. ${ }^{[12]}$ In this latter study, both the baseline and treatment-associated changes in levels of plasma HIV RNA were independent predictors of clinical progression to AIDS or death, whereas CD4+ counts were not.

\section{Antiretroviral Treatment}

A number of antiretroviral drugs have been approved by the US Food and Drug Administration (FDA) and are listed in table II. An international panel of experts recommended that antiretroviral treatment should be started for all symptomatic HIV-infected patients and most asymptomatic patients with CD $4+$ counts of $<500 / \mathrm{mm}^{3}$. ${ }^{[13]}$ However, they acknowledged a paucity of prospective data concerning the management of patients with stable CD4+ counts of 350 to $500 / \mathrm{mm}^{3}$ with low or undetectable plasma HIV RNA levels. Further, the only randomised, placebo-controlled trial conducted to date in asymptomatic patients with initial CD4+ counts of $>500 / \mathrm{mm}^{3}$ showed no benefit to early treatment. ${ }^{[14]}$

Despite these limitations, the 3 basic principles of antiretroviral treatment for asymptomatic HIVinfected patients are:
- Treatment should be considered for all patients with HIV infection. It should be strongly recommended for those with HIV RNA levels of $>10000$ copies $/ \mathrm{mm}^{3}$, with rising levels of HIV RNA or with falling CD4+ count.

- For those with low or undetectable plasma levels of HIV RNA and stable CD4+ counts, careful monitoring at 3- to 4-month intervals without antiretroviral treatment should be considered.

- Response to antiretroviral treatment should be monitored by quantifying plasma HIV RNA for 4 to 6 weeks after initiation of treatment.

\subsection{Monotherapy with Antiretroviral Drugs}

Monotherapy with antiretroviral drugs at best provides transient clinical benefit for asymptomatic patients. Zidovudine is the oldest and best evaluated of the antiretroviral drugs. The effects of zidovudine monotherapy have been studied in asymptomatic patients at varying stages of disease. For example, more than 1600 patients with initial CD $4+$ counts of $>500 / \mathrm{mm}^{3}$ were entered into a randomised ACTG trial comparing $500 \mathrm{mg} /$ day, $1500 \mathrm{mg} /$ day or delayed zidovudine therapy ${ }^{[14]} \mathrm{Af}-$ ter a mean of 4.8 years of follow-up, there were no significant differences in the AIDS-free or overall survival of the treatment groups.

Table II. Antiretroviral drugs

\begin{tabular}{|c|c|c|c|}
\hline Drug & Generic name & $\begin{array}{l}\text { Oral dose } \\
(\mathrm{mg})\end{array}$ & $\begin{array}{l}\text { Estimated daily } \\
\text { cost, } 1996 \text { (\$US) }\end{array}$ \\
\hline \multicolumn{4}{|c|}{ Nucleoside analogues } \\
\hline$A Z T$ & Zidovudine & 200 tid & 9.30 \\
\hline ddl & Didanosine & 200 bid & 6.00 \\
\hline$d d C$ & Zalcitabine & $0.75 q 8 \mathrm{~h}$ & 6.90 \\
\hline $\mathrm{d} 4 \mathrm{~T}$ & Stavudine & 40 bid & 8.10 \\
\hline ЗТC & Lamivudine & 150 bid & 7.40 \\
\hline \multicolumn{4}{|c|}{ Protease inhibitors } \\
\hline SQV & Saquinavir & 600 tid & 19.00 \\
\hline RTV & Ritonavir & 600 bid & 22.00 \\
\hline IND & Indinavir & 800 tid & 15.00 \\
\hline \multicolumn{4}{|c|}{ Non-nucleoside reverse transcriptase inhibitor } \\
\hline NVP & Nevirapine & 200 bid & 5.22 \\
\hline $\begin{array}{l}\text { Abbre } \\
\text { daily. }\end{array}$ & s: bid = twice de & $\mathrm{q} 8 \mathrm{~h}=\mathrm{ever}$ & hours; tid $=3$ times \\
\hline
\end{tabular}


Zidovudine monotherapy has also been evaluated in patients with more advanced HIV infection. VA, ACTG protocol 019 and Concorde studies provide examples of the transient benefits of zidovudine monotherapy. ${ }^{[15-18]}$ In the VACooperative Studies trial, early versus delayed zidovudine treatment reduced the risk of progression to AIDS but did not improve survival. ${ }^{[15]}$ In the ACTG protocol 019 study, zidovudine compared with placebo treatment provided initial protection against clinical progression but no survival benefit. [16] However, the protection against clinical progression in this study diminished after 2 years of follow-up. ${ }^{[17]}$ In Concorde, neither progression to AIDS nor survival was improved in the patients who received early compared with deferred zidovudine treatment. ${ }^{[18]}$

Monotherapy with didanosine has also been extensively studied. ACTG protocol 116A was a randomised trial of zidovudine compared with high or low dose didanosine monotherapy for patients who had received little or no prior zidovudine therapy. ${ }^{[19]}$ It showed a reduced rate of clinical progression among the patients randomised to zidovudine with no prior zidovudine therapy. It also showed a reduced rate of clinical progression among the patients randomised to didanosine who had received as little as 8 to 16 weeks of prior zidovudine therapy.

Three other studies confirmed these latter results. In ACTG protocol 116B/117, patients who had received at least 16 weeks of zidovudine treatment were randomly assigned to continue on zidovudine or to receive high or low dose didanosine. ${ }^{[20]}$ The patients assigned to low dose didanosine had significantly reduced risk of progression compared with those remaining on zidovudine.

The Canadian Network conducted a randomised trial comparing zidovudine with didanosine in patients with 6 months of prior zidovudine experience and CD4+ counts of 200 to $500 / \mathrm{mm}^{3}{ }^{[21]}$ There were significantly fewer AIDS events in the group assigned to didanosine.

Finally, a multicentre, randomised, double-blind study compared continued zidovudine with didano- sine therapy in patients who received zidovudine for at least 6 months and had signs of clinical deterioration. ${ }^{[22]}$ There was a reduced risk of clinical progression to a new AIDS event or death among the group assigned to didanosine. These studies confirm a time-limited benefit to zidovudine monotherapy as well as a benefit to a switch to didanosine.

Experience with other nucleoside analogues as monotherapy for HIV infection is more limited. For example, Fischl et al. ${ }^{[23]}$ reported results of a randomised trial comparing continued zidovudine with zalcitabine (ddC) in patients who had experienced a first episode of PCP or advanced AIDS and had tolerated a minimum of 48 weeks of zidovudine treatment. No differences were observed in survival or clinical end-points. The Community Program for Clinical Research on AIDS (CPCRA) conducted a randomised trial comparing didanosine with zalcitabine in patients who progressed on or were intolerant of zidovudine treatment. ${ }^{[24]}$ Progression or death occurred in 93.3 and $87.8 \%$ of the patients randomised to didanosine and zalcitabine, respectively $(\mathrm{p}=0.15)$.

Fischl et al. ${ }^{[25]}$ reported the results of a randomised trial comparing continued zidovudine, zalcitabine or the combination of zidovudine plus zalcitabine in patients who had tolerated at least 6 months of zidovudine treatment. Patients were stratified on the basis of their prerandomisation CD4+ counts. There were no overall outcome differences between the treatment groups. However, in the subset of patients with the highest pretreatment CD4+ count, there were fewer progressions or deaths in those randomised to the combination of zidovudine plus zalcitabine compared with zidovudine treatment. These data support the use of a combination of antiretroviral drugs, more fully discussed in section 2.2 .

\subsection{Combination Therapy with Antiretroviral Drugs}

Combinations of antiretroviral drugs to treat HIV infection are advocated for several reasons. 
- HIV replicates in patients at an enormous rate. As noted above, infected patients produce more than 1 billion $\left(10^{9}\right)$ HIV virions daily during the steady-state of infection.

- Most of the nucleoside analogues have relatively modest antiretroviral activity when used alone, reducing viral loads by $<1$ log even during early stages of treatment. ${ }^{[11,12,26]}$

- Monotherapy results in the selection of resistant HIV strains and uninhibited replication of these is associated with clinical deterioration. ${ }^{[27-29]}$

- The clinical benefits of early monotherapy with antiretroviral drugs, such as zidovudine, have proven to be transient. ${ }^{[15-18]}$

\subsubsection{Nucleoside Analogues}

A number of antiretroviral drug combinations have been evaluated in HIV-infected patients. ACTG protocol $175^{[30]}$ and the European/Australian Delta Trials ${ }^{[31]}$ compared the risks of clinical disease (progression to AIDS or death) in patients randomised to receive the combinations of zidovudine plus didanosine or zidovudine plus zalcitabine with zidovudine monotherapy. The patients enrolled in ACTG 175 had CD4+ counts between 200 and 500 cells $/ \mathrm{mm}^{3}$, while those in the Delta Trial had CD $4+$ counts of $<350 / \mathrm{mm}^{3}$.

In both studies, patients randomised to the zidovudine plus didanosine combination were significantly less likely to progress than those randomised to zidovudine monotherapy. In ACTG 175, this benefit was observed among all of the subsets, whereas in the Delta Trial, involving more advanced patients, the benefit was observed only among those patients who were initially zidovudine-naive. The patients randomised to zidovudine plus zalcitabine also received a marginal benefit. In ACTG 175 , one group of patients was randomised to didanosine monotherapy; this group did as well as those randomised to zidovudine plus didanosine.

A CPCRA trial also compared the combinations of zidovudine plus didanosine, zidovudine plus zalcitabine and zidovudine monotherapy. ${ }^{[32]}$ In this study, patients with AIDS or with CD4+ counts of $<200 / \mathrm{mm}^{3}$ were randomised to the 3 treatment arms. Although the overall AIDS-free survival and survival were essentially the same in the treatment arms, the patients who had not received prior zidovudine treatment and were randomised to the combination treatments appeared to benefit.

The data from these 3 trials suggest that a combination of two new (previously unused) antiretrovirals may be required for clinical benefit in patients with advanced HIV infection, whereas didanosine monotherapy may be acceptable in less advanced patients.

Lamivudine (3TC) is a potent reverse transcriptase inhibitor which has a broad range of activity against HIV. Mutations at codon 184 of the polymerase gene occur rapidly in patients exposed to this agent and confer resistance to it. ${ }^{[33]}$ These strains, however, remain sensitive to zidovudine. ${ }^{[34]}$ A double-blind study was conducted in HIV-infected patients with CD4+ counts of 200 to 500 cells $/ \mathrm{mm}^{3}$ already on treatment with zidovudine. ${ }^{[35]}$ The patients were randomly assigned to receive treatment with zidovudine alone, lamivudine alone or a combination of zidovudine plus lamivudine. The combination was well tolerated by the study participants. Further, the patients on combined treatment had a striking increase in their CD4+ counts and reduction in their plasma HIV RNA levels which persisted over both the initial period and an extended period of 52 weeks of blinded follow-up. ${ }^{[36]}$

Another study compared the combinations of zidovudine plus lamivudine [given in low $150 \mathrm{mg}$ twice daily) or high (300mg orally twice daily) doses] with zidovudine plus zalcitabine in patients already on zidovudine with CD4+ counts of 100 to 300 cells $/ \mathrm{mm}^{3}{ }^{3}{ }^{[37]}$ The patients randomised to the zidovudine plus lamivudine combinations had an increase in their CD4+ counts, but HIV RNA levels were similar for all groups. Further, the low doses of lamivudine were better tolerated. However, there were too few end-points to draw any conclusions about the clinical benefits of the combination of zidovudine plus lamivudine in either of these studies. 


\subsubsection{Non-Nucleoside Reverse Transcriptase Inhibitors}

Nevirapine is the first non-nucleoside reverse transcriptase inhibitor to receive conditional approval for use by the FDA. A pilot study in asymptomatic patients showed that nevirapine monotherapy resulted in rapid selection of resistant strains. ${ }^{[38]}$ A study comparing the combination of nevirapine plus zidovudine plus didanosine with the combination of zidovudine plus didanosine was recently reported. ${ }^{[39]}$ After 48 weeks of treatment, patients assigned to the triple combination had a higher mean CD4+ count and a lower mean HIV RNA concentration than did those assigned to the double combination. However, the patients assigned to receive nevirapine also experienced more severe rashes, and there were no differences in the risks for disease progression in the small groups of patients studied. On the basis of these findings, enthusiasm for this combination must be very limited.

\subsubsection{Protease Inhibitors}

Saquinavir was the first of the protease inhibitors to receive conditional approval for use by the FDA. It was evaluated by the AIDS Clinical Trials Group (ACTG) in Protocol 229. In this study, patients with prior zidovudine experience were randomly assigned to receive zidovudine plus zalcitabine, zidovudine plus saquinavir, or the triple combination of zidovudine, zalcitabine and saquinavir. ${ }^{[40]}$ After 48 weeks of therapy, $51 \%$ of the patients on triple therapy, $34 \%$ on zidovudine plus zalcitabine and $33 \%$ on zidovudine plus saquinavir had CD4+ counts above baseline $(p=0.0013)$. On triple therapy, HIV RNA titres initially fell by 0.8 $\log$, while they fell $0.4 \operatorname{logs}$ on the double therapy combinations $(p<0.001)$. Again, clinical evidence of efficacy is lacking.

Ritonavir is a potent HIV protease inhibitor which has high oral bioavailability. Preliminary clinical results of a multicentre, double-blind trial of ritonavir were recently reported. ${ }^{[41]}$ HIV infected patients with $\mathrm{CD} 4+$ counts of 100 cells $/ \mathrm{mm}^{3}$ were randomly assigned to receive ritonavir in a dose of $600 \mathrm{mg}$ orally twice daily or placebo, Other anti- retroviral drugs were permitted. The end-points of the study were AIDS-defining events and death. Adverse events were observed in $17 \%$ of the patients receiving ritonavir and $6 \%$ of the placebo recipients. These included nausea, vomiting, weakness and diarrhoea. In a median of 6.1 months of follow-up, there were $26(4.8 \%)$ deaths in 543 patients assigned to ritonavir compared with 46 (8.4\%) deaths in 547 patients assigned to placebo $(\mathrm{p}=0.02)$. AIDS or death were observed in 69 $(12.7 \%)$ and $149(27.3 \%)$ of the patients assigned to ritonavir and placebo, respectively $(\mathrm{p}<0.001)$. There was a surprisingly large number of Kaposi's sarcoma AIDS-defining diagnoses in the placebo group. However, these data suggest that ritonavir treatment is associated with a substantial benefit in preventing morbidity and mortality in patients with advanced HIV infection.

Indinavir is another potent HIV protease inhibitor with high oral bioavailability. Nephrolithiasis is a significant side effect of treatment with this drug. A double-blind trial of indinavir in patients with 6 months of zidovudine treatment, CD4+ counts of 50 to 400 cells $/ \mathrm{mm}^{3}$ and plasma HIV RNA levels of $>20000$ copies $/ \mathrm{ml}$ was recently reported. ${ }^{[42]}$ A total of 97 patients were randomly assigned to receive indinavir alone, zidovudine plus lamivudine or indinavir plus zidovudine plus lamivudine. Plasma HIV RNA levels fell to below the lower limit of detection ( $<500$ copies $/ \mathrm{ml}$ ) in 88 and $84 \%$ of the patients on triple therapy and 41 and $44 \%$ of the patients on indinavir alone at 12 and 24 weeks of treatment, respectively. In contrast, undetectable levels of plasma HIV RNA were observed in only $8 \%$ of the patients on zidovudine and lamivudine at 12 weeks and none at 24 weeks of therapy.

These data suggest that the combination of indinavir plus zidovudine plus lamivudine is the most potent combination of antiretroviral drugs tested to date. A trial of indinavir with clinical endpoints is now in progress.

Finally, preliminary results have been reported of a trial in which patients received a combination of 2 protease inhibitors, saquinavir plus ritonavir. ${ }^{[43]}$ 
Table III. Treatment ard prophylaxis for infections in patients with asymptomatic HIV infection

\begin{tabular}{|c|c|c|c|}
\hline Infection & Indication & Preferred therapy/prophylaxis & Alternative \\
\hline Tuberculosis & Positive PPD & INH $300 \mathrm{mg}$ orally daily & $\begin{array}{l}\text { Rifampicin (rifampin); pyrazinamide + ethambutol; } \\
\text { pyrazinamide + fluoroquinalone }\end{array}$ \\
\hline PCP & $\mathrm{CD} 4+\leq 200$ & Daily TMP-SMX DS & Dapsone; dapsone + trimethoprim; aerosol pentamidine \\
\hline Toxoplasmosis & $\mathrm{CD} 4+<100$ & Daily TMP-SMX DS & Dapsone + pyrimethamine \\
\hline DMAC & $\mathrm{CD} 4+<75$ & Azithromycin $1200 \mathrm{mg} / \mathrm{wk}$ & Clarithromycin $500 \mathrm{mg}$ bid; rifabutin $300 \mathrm{mg}$ od \\
\hline CMV & $\mathrm{CD} 4+<50$ & None & Oral ganciclovir $1 \mathrm{~g} P O$ tid \\
\hline Cryptococcosis & $\mathrm{CD} 4+<50$ & None & Fluconazole $200 \mathrm{mg} /$ day PO \\
\hline S. pneumoniae & All $\mathrm{HIV}_{+}$ & Pneumococcal vaccine & \\
\hline
\end{tabular}

Abbreviations: bid = twice daily; $\mathrm{CMV}=$ cytomegalovirus; $\mathrm{DMAC}=$ disseminated Mycobacterium avium complex; od $=$ once daily; $\mathrm{INH}=$ isoniazid; $\mathrm{PCP}=$ Pneumocystis carinii pneumonia; $\mathrm{PPD}=$ purified protein derivative; $\mathrm{PZA}=$ pyrazinamide; $\mathrm{PO}=$ orally; tid $=3$ times daily; TMP-SMX DS = cotrimoxazole (trimethoprim plus sulfamethoxazole), double strength; TMP = trimethoprim.

In this study, patients with CD4+ counts of 100 to $500 / \mathrm{mm}^{3}$ were randomly assigned to 1 of 4 regimens combining these drugs. Median plasma HIV RNA levels fell 2 to 3 logs from baseline levels with each of the regimens tested, and they were generally well tolerated. Long term follow-up and clinical studies of protease inhibitor combinations are pending.

The above data suggest that combinations of antiretroviral drugs are appropriate for most patients receiving treatment. didanosine is the only antiretroviral agent which can be used alone, and this should be selected only in patients with less advanced disease, such as those with CD4+ counts of 350 to $500 / \mathrm{mm}^{3}$ and a plasma HIV viral load of $<10000$. Although optimal regimens for patients with more advanced disease have not been established by comparative clinical trials, combinations including a protease inhibitor with one or more new nucleoside analogues or a combination of protease inhibitors seem most promising.

\section{Additional Treatment and Prophylaxis}

Infections caused by other micro-organisms produce much of the morbidity and mortality in HIV-infected patients. Clinically active infections are generally associated with symptoms. Their treatment, therefore, is beyond the scope of this article. Many organisms, however, cause latent infections which can be detected and treated before symptoms occur. Others can be prevented by appropriate immunisations or use of antimicrobial agents. Some of these are shown in table III and discussed below.

\subsection{Tuberculosis}

Clinically active Mycobacterium tuberculosis infection occurs at all stages of HIV disease. The tuberculin skin test is the most sensitive means of detecting asymptomatic, subclinical $M$. tuberculosis infection. Induration of $5 \mathrm{~mm}$ is considered to be positive in HIV-infected patients, and $8 \%$ per year of the PPD-positive, HIV-infected patients develop clinically active disease. ${ }^{[44]}$ A case-control study showed that clinical tuberculosis also accelerates the downhill course of HIV infection. ${ }^{\text {[45] }}$

A year of preventive treatment with isoniazid $300 \mathrm{mg}$ orally daily plus pyridoxine $50 \mathrm{mg}$ orally daily is recommended for tuberculin-positive HIVinfected patients with no evidence of clinical disease. ${ }^{[46]}$ For those known to have been exposed to patients with multi-drug-resistant $M$. tuberculosis infections, reasonable alternatives are treatment with rifampicin (rifampin) $600 \mathrm{mg}$ orally daily, pyrazinamide plus ethambutol or pyrazinamide plus a fluroquinolone.

\subsection{Pneumocystis carinii Pneumonia}

PCP is the most common life-threatening opportunistic infection that occurs in HIV-infected patients. Most PCP infections occur in patients with CD4+ counts of $200 / \mathrm{mm}^{3} \cdot{ }^{[47]}$ Such patients should be given prophylaxis. Randomised, controlled clinical trials showed that cotrimoxazole is the most 
effective agent for prophylaxis against both primary ${ }^{[48]}$ and recurrent ${ }^{[49]}$ PCP infection and will prevent most disease if used properly. However, allergy to or intolerance of cotrimoxazole is common among HIV-infected patients. Therefore, alternatives may be required, such as dapsone, dapsone plus trimethoprim, or aerosolised pentamidine. ${ }^{[50]}$

\subsection{Toxoplasma Encephalitis}

Toxoplasma gondii is an obligate intracellular parasite which causes encephalitis and disseminated disease in HIV-infected patients. Most infections occur as a result of reactivation of latent infection in $T$. gondii seropositive, HIV-infected patients with CD4+ counts of $100 / \mathrm{mm}^{3}{ }^{3}{ }^{51]}$ Such patients should be considered for prophylaxis, even when asymptomatic. Fortunately, cotrimoxazole, one of the agents used to prevent PCP infection, appears to be effective against $T$. gondii also. ${ }^{[52]}$ The combination of dapsone and pyrimethamine is an alternative in those who are allergic to or cannot tolerate cotrimoxazole.

\subsection{Disseminated Mycobacterium avium Complex Disease}

DMAC is the most common opportunistic bacterial infection occurring in patients with HIV infection. For example, Nightingale et al. followed over 1000 patients after a clinical diagnosis of AIDS and demonstrated, by monthly lysis-centrifugation of blood cultures, that the incidences of MAC bacteraemia were $21 \pm 2 \%$ and $41 \pm 3 \%$ at 1 and 2 years, respectively. ${ }^{[53]}$ Most DMAC infections occur at late stages of HIV infection. In the study cited above, the 1-year incidence of MAC bacteraemia was $39,30,20,15,8,3$ and $1 \%$ for patients with CD4+ counts of 0 to 9,10 to 19,20 to 49,40 to 59,60 to 99,100 to 199 and 200 to 499 cells $/ \mathrm{mm}^{3}$, respectively. The median survival of patients following diagnosis of MAC bacteraemia was 134 days.

Three drugs have been shown to be effective as prophylaxis against DMAC infections in randomised, controlled clinical trials. These are rifabutin, ${ }^{[54]}$ clarithromycin ${ }^{[55]}$ and azithromycin. ${ }^{[56]} \mathrm{Un}$ - fortunately, breakthrough infections occur with these agents. The DMAC isolates obtained from patients receiving rifabutin prophylaxis are generally sensitive to rifabutin, whereas those isolated from patients on either clarithromycin or azithromycin prophylaxis are generally resistant to both clarithromycin and azithromycin. At present, DMAC prophylaxis is recommended for patients with CD4+ counts of $<75 / \mathrm{mm}^{3}{ }^{[57]}$ However, the agents used for prophylaxis may interact with antiretroviral drugs such as protease inhibitors. Since it is not yet known whether the virological and immunological improvement which occurs on treatment will reduce the risk of DMAC infections, many experts defer DMAC prophylaxis until combinations of antiretroviral drugs fail or are not tolerated.

\subsection{Cytomegalovirus Disease}

CMV disease is demonstrated clinically or by autopsy in most patients with HIV infection. The most common manifestation of CMV in HIVinfected patients is retinitis which can lead to blindness. CMV also causes ulcerative gastrointestinal disease, encephalitis, polyradiculitis, pneumonia and adrenalitis.

One randomised, placebo-controlled trial in HIV-infected patients showed that oral ganciclovir significantly reduced the incidence of CMV disease but did not improve overall survival ${ }^{[58]}$ However, a second trial, conducted by the CPCRA, showed no differences in the incidence of CMV disease in the placebo and ganciclovir recipients. ${ }^{59]}$ There were significantly more adverse events in the patients randomised to ganciclovir than in the placebo recipients.

Analysis suggested that in 1996 it cost \$US 144000 to prevent each case of CMV disease and \$US 15000 to extend disease-free survival by 1 month. ${ }^{[60]}$ On the basis of these data, most experts do not recommend the use of oral ganciclovir for primary CMV prophylaxis at this time.

\subsection{Invasive Fungal Infections}

Cryptococcus neoformans and Candida spp. cause invasive disease in patients with advanced HIV in- 
fection. Candida oesophagitis generally follows a more localised oral infection which is likely to produce symptoms. Cryptococcosis, in contrast, frequently occurs in previously asymptomatic patients. A randomised trial compared the efficacy of oral fluconazole with clotrimazole troches as prophylaxis against invasive fungal infection. ${ }^{[61]} \mathrm{Pa}$ tients randomised to the latter had significantly increased risk of cryptococcosis (relative hazard 8.5; $95 \%$ CI 1.9 to 37.6 ). Most of the cryptococcal infections occurred in patients with baseline CD4+ counts of $<50 / \mathrm{mm}^{3}$, and $78 \%$ of them in patients whose most recent CD4+ count was below this level. Despite these data, prophylaxis to prevent cryptococcosis is not recommended because of the relatively low incidence of this infection and the expense of prophylactic treatment. In the randomised trial described above, it was estimated that 11 756 doses of fluconazole were administered at a cost of over $\$$ US 100000 for each case of cryptococcosis prevented.

\subsection{Streptococcus pneumoniae Infections}

S. pneumoniae is the most common cause of community-acquired bacterial pneumonia in HIVinfected patients. ${ }^{[62]}$ Though they are not associated with increased mortality, bacteraemic pneumococcal infections occur far more commonly in HIV-infected than in non-HIV-infected patients. ${ }^{[63]}$ Many of these infections can be prevented by use of the 23-valent pneumococcal capsular polysaccharide vaccine. Pneumococcal vaccine failures have been reported in HIV-infected patients. ${ }^{[64]}$ However, asymptomatic individuals with relatively intact immune systems appear to respond normally to the pneumococcal vaccine. ${ }^{[65]}$ The Advisory Committee on Immunisation Practices to the US Public Health System recommends the use of pneumococcal vaccine for all $\mathrm{HIV}$-infected patients. ${ }^{[66]}$

\section{Conclusions}

Patients are asymptomatic during most of the initial phases of HIV infection. The virus, however, is actively replicating during this period. Manage- ment of asymptomatic HIV infection involves recognition of infection and counselling to prevent its further spread. When HIV infection has been confirmed, assessment consists of a careful history, physical examination and laboratory studies. The latter includes serological tests for past exposure to infectious agents such as syphilis, hepatitis viruses, cytomegalovirus and toxoplasmosis. A PPD skin test also should be performed.

Immunological and virological status of the patient are assessed by quantifying CD4+ count and plasma HIV RNA concentrations. With the aid of these data, a decision on use of antiretroviral therapy can be made. Didanosine is the only currently acceptable monotherapy. Combinations of antiretroviral drugs which have undergone evaluation include zidovudine plus didanosine, zidovudine plus zalcitabine, zidovudine plus lamivudine, didanosine plus lamivudine, zidovudine plus didanosine plus nevirapine, zidovudine plus saquinavir, zidovudine plus zalcitabine plus saquinavir, zidovudine plus ritonavir, zidovudine plus lamivudine plus indinavir, and ritonavir plus saquinavir. The optimum combination and timing of treatment have not been defined. Future research should clarify these.

In addition to antiretroviral therapy, treatment for subclinical infections and prophylaxis should be administered. These include:

- Treatment of all PPD positive patients with isoniazid $300 \mathrm{mg} /$ day orally for 1 year.

- Prophylaxis against PCP for all patients with CD $4+$ counts $<200 / \mathrm{mm}^{3}$ with trimethoprim plus sulfamethoxazole one double-strength tablet daily, dapsone or dapsone plus trimethoprim.

- Prophylaxis against toxoplasmosis in patients with CD4+ counts $<100 / \mathrm{mm}^{3}$ with trimethoprim plus sulfamethoxazole or with dapsone plus pyrimethamine.

- Prophylaxis against DMAC disease in patients with CD $4+$ counts $<75 / \mathrm{mm}^{3}$ with azithromycin $1200 \mathrm{mg}$ orally once weekly, clarithromycin $500 \mathrm{mg}$ orally twice daily, or rifabutin $300 \mathrm{mg}$ /day orally.

- Prophylaxis against $S$. pnuemoniae pnuemonia and bacteraemia by administration of the 23valent pneumococcal vaccine. 
Future research also should define more effective means of preventing or suppressing the host of infections that complicate HIV disease.

\section{References}

1. Quinn TC. Global burden of the HIV pandemic. Lancet 1996; 348: 99-106

2. Wei $X$, Gosh SK, Taylor ME, et al. Viral dynamics in human immunodeficiency virus type 1 infection. Nature $1995 ; 373$ $117-22$

3. Ho DD, Neuman AU, Perelson AS, et al. Rapid turnover of plama virions and CD4 lymphocytes in HIV-1 infection. Nature 1995; 373: 123-6

4. Fahey JL, Taylor JMG, Detels R, et al. The prognostic value of cellular and serologic markers in infection with human immunodeficiency virus type 1 . N Engl J Med 1990; 322: 166-72

5. Kaplan JE, Masur H, Holmes KK, et al. USPHS/IDSA guidelines for the prevention of opportunistic infections in persons infected with human immunodeficiency virus: an overview. Clin Infect Dis 1995; 21 Suppl. 1: S12-31

6. Saag MS, Holodoniy M, Kuritzkes DR, et al. HIV viral load markers in clinical practice. Nature Med 1996; 2: 625-9

7. Mellors JW, Kingsley LA, Rinaldo CR, et al. Quantitation of HIV-1 RNA in plasma predicts outcome after seroconversion. Ann Intern Med 1995; 122: 573-9

8. Mellors JW, Rinaldo CR, Gupta P, et al. Prognosis in HIV-1 infection predicted by the quantity of virus in plasma. Science 1996; 272: 1167-70

9. O'Brien TR, Blattner WA, Waters D, et al. Serum HIV-I RNA levels and time to development of AIDS in the multicenter hemophilia cohort study. JAMA 1996; 276: 106-10

10. Welles SL, Jackson JB, Yen-Lieberman B, et al. Prognostic value of plasma human immunodeficiency virus type 1 (HIV1) RNA levels in patients with advanced HIV-1 disease and with little or no prior zidovudine therapy. J Infect Dis 1996 ; 174: 696-703

11. O'Brien WA, Hartigan PM, Martin D, et al. Changes in plasma HIV-1 RNA and CD4+ lymphocyte count relative to treatment and progression to AIDS. N Engl J Med 1996; 334: 426-31

12. Katzenstein DA, Hammer SM, Hughes MD, et al. The relation of virologic and immunologic markers to clinical outcomes after nucleoside therapy in HIV-infected adults with 200 to 500 CD4 cells per cubic millimeter. N Engl J Med 1996; 336 : $1091-8$

13. Carpenter CJ, Fischl MA, Hammer SM, et al. Consensus statement: antiretroviral therapy for HIV infection in 1996. Recommendations of an international panel. JAMA 1996; 276: $146-54$

14. Volberding PA, Lagakos SW, Grimes JM, et al. A comparison of immediate with deferred zidovudine therapy for asymptomatic HIV-infected adults with CD4 cell counts of 500 or more per cubic millimeter. N Engl J Med 1995; 333: 401-7

15. Hamilton JD, Hartigan PM, Simberkoff MS, et al. A controlled trial of early versus later zidovudine in symptomatic human immunodeficiency virus infection: results of a Veterans Affairs Cooperative Study. N Engl J Med 1992; 326; 437-43

16. Volberding PA, Lagakos SW, Koch MA, et al. Zidovudine in asymptomatic human immunodeficiency virus infection: a controlled trial in persons with fewer than $500 \mathrm{CD} 4$-positive cells per cubic millimeter. N Engl J Med 1990; 322: $941-9$
17. Volberding PA, Lagakos SW, Grimes JM, et al. The duration of zidovudine benefit in persons with asymptomatic HIV infection: prolonged evaluation of protocol 019 of the AIDS Clinical Trials Group. JAMA 1994; 272: 437-42

18. Concorde Coordinating Committee. Concorde MRC/ANRS randomized double-blind controlled trail of immediate and deferred zidovudine in symptom-free HIV infection. Lancet 1994; 343: 871-81

19. Dolin R, Amato DA, Fischl MA, et al. Zidovudine compared with didanosine in patients with advanced HIV type 1 infection and little or no previous experience with zidovudine. Arch Intern Med 1995; 155: 96 I-74

20. Kahn JO, Lagakos SW, Richman DD, et al. A controlled trial comparing continued zidovudine with didanosine in human immunodeficiency virus infection. N Engl J Med 1992; 327 $581-7$

21. Montaner JSG, Schechter MT, Rachlis A, et al. Didanosine compared with continued zidovudine therapy for HIVinfected patients with 200 to $500 \mathrm{CD} 4 \mathrm{cells} / \mathrm{mm}^{3}$ : a doubleblind, randomized, controlled trial. Ann Intern Med 1995; 123: $561-71$

22. Spruance SL, Pavia AT, Peterson D, et al. Didanosine compared with continuation of zidovudine in HIV-infected patients with signs of clinical deterioration while receiving zidovudine: a randomized, double-blind clinical trial. Ann Intern Med 1994; 120: 360-8

23. Fischl MA, Olson RM, Follansbee SE, et al. Zalcitabine compared with zidovudine in patients with advanced HIV-1 infection who received previous zidovudine therapy. Ann Intern Med 1993; 118: 762-9

24. Abrams DI, Goldman AI, Launer C, et al. A comparative trial of didanosine or zalcitabine after treatment with zidovuine in patients with human immunodeficiency virus infection. N Engl J Med 1994; 330: 657-62

25. Fischl MA, Stanley K, Arduino JM, et al. Combination and monotherapy with zidovudine and zalcitabine in patients with advanced HIV disease. Ann Intern Med 1995; 122: 24-32

26. Cao Y, Ho DD, Todd JJ, et al. Clinical evaluation of branched DNA signal amplification for quantifying HIV type 1 in human plasma. AIDS Res Hum Retroviruses 1995; 11: 353-61

27. Larder BA, Darby G, Richmann DD. HIV with reduced sensitivity to zidvudine (AZT) isolated during prolonged therapy. Science 1989; 243 : 1731-4

28. Johnson VA. Nucleoside reverse transcriptase inhibitors and resistance of human immunodeficiency virus type 1 . J Infect Dis 1995; 171 Suppl. 2: S140-9

29. Markowitz M, Mo H, Kempf DJ, et al. Selection and analysis of human immunodeficiency virus type 1 variants with increased resistance to ABT-538, a novel protease inhibitor. J Virol 1995; 69: 701-6

30. Hammer SM, Katzenstein DA, Hughes MD, et al. A trial comparing nucleoside montherapy with combination therapy in HIV infected adults with CD 4 cell counts from 200 to 500 per cubic millimeter. N Engl J Med 1996; 335: 1081-90

31. Delta Coordinating Committee. Delta: a randomized doubleblind controlled trial comparing combinations of zidovudine plus didansosine or zalcitabine with zidovudine alone in HIVinfected individuals. Lancet 1996; 348: 283-91

32. Saravolatz LD, Winslow DL, Collins G, et al. Zidovudine alone or in combination with didanosine or zalcitabine in HIV infected patients with the acquired immunodeficiency syndrome or fewer than $100 \mathrm{CD} 4$ cells per cubic millimeter. $\mathrm{N}$ Engl J Med 1996; 335: 1099-106 
33. Boucher CA, Cammack N, Schipper P, et al. High level resistance to () enantiomeric 2-deoxy-3-thiacytidine in vitro is due to one amino acid substitution in the catalytic site of human immunodeficiency virus type 1 reverse transcriptase. Antimicrob Agents Chemother 1993; 37: $2231-4$

34. Schinazi RF, Lloyd RM, Nguyen MH, et al. Characterization of human immunodeficiency viruses resistant to oxanthiolanecytosine nucleosides. Antimicrob Agents Chemother 1993; 37: $875-81$

35. Eron JJ, Benoit SI, Jemsek J, et al. Treatment with lamivudine, zidovudine, or both in HIV-positive patients with 200 to 500 CD4+ cells per cubic millimeter. N Engl J Med 1995; 333 : $1662-9$

36. Eron JJ, Quinn JB, Hill-Price S, et al. 52 week follow-up of NUCA 3001: 3TC, zidovudine or both in the treatment of HIV-positive patients with CD4 cell counts of $200-500$ cells $/ \mathrm{mm}^{3}$. Presented at the 3rd Conference on Retroviruses and Opportunistic Infections; 1996 Jan 29-Feb 1; Washington, DC: The American Society for Microbiology, 1996

37. Bartlett JA, Benoit SL, Johnson VA, et al. Lamivudine plus zidovudine compared with zalcitabine plus zidovudine in patients with HIV infection: a randomized, double-blind, placebo-controlled trial. Ann Intern Med 1996; 125: 161-72

38. Havlir D, McLaughlin MM, Richman DD. A pilot study to evaluate the development of resistance to nevirapine in asymptomatic human immunodeficiency virus-infected patients with CD4 cell counts of $>500 / \mathrm{mm}^{3}$ : AIDS Clinical Trials Group Protocol 208. J Infect Dis 1995; 172: 1379-83

39. D'Aquila RT, Hughes MD, Johnson VA, et al. Nevirapine, zidovudine, and didanosine compared with zidovudine and didanosine in patients with HIV-1 infection: a randomized, double-blind, placebo-controlled trial. Ann Intern Med 1996; 124: $1019-30$

40. Collier AC, Coombs RW, Schoenfeld DA, et al. Treatment of human immunodeficiency virus infection with saquinavir, zidovudine, and zalcitabine. N Engl J Med 1996; 334: 1011-7

41. Cameron B, Heath-Chiozzi M, Kravcik S, et al. Prolongation of life and prevention of AIDS in advanced HIV immunodeficiency with ritonavir. Presented at the 3rd Conference on Retroviruses and Opportunistic Infections; 1996 Jan 29-Feb 1; Washington, DC: The American Society for Microbiology, 1996

42. Gulick R, Mellors J, Havlir D, et al. Potent and sustained antiretroviral activity of indinavir in combination with zidovudine and lamivudine. Presented at the 3rd Conference on Retroviruses and Opportunistic Infections; 1996 Jan 29-Feb 1; Washington, DC: The American Society for Microbiology, 1996

43. Cohen C, Sun E, Cameron D, et al. Ritonavir-saquinavir combination treatment in HIV-infected patients [abstract no. LB7b]. Presented at the 36th Interscience Conference on Antimicrobial Agents and Chemotherapy; 1996 Sep 15-18; New Orleans. Washington, DC: The American Society for Microbiology, 1996

44. Selwyn PA, Hartel D, Lewis VA, et al. A prospective study of the risk of tuberculosis among intravenous drug users with human immunodeficiency virus infection. $N$ Engl $J$ Med 1989; 320: $545-50$

45. Whalen C, Horsburgh CR, Hom D, et al. Accelerated course of human immunodeficiency virus infection after tuberculosis. Am J Respir Crit Care 1995; 151: 129-35

46. Castro $\mathrm{KG}$. Tuberculosis as an opportunistic disease in persons infected with human immunodeficiency virus. Clin Infect Dis 1995; 21 Suppl. 1: S66-71
47. Phair J, Muñoz A, Detels R, et al. The risk of Pneumocystis carinii pneumonia among men infected with human immunodeficiency virus type 1. N Engl J Med 1990; 322: 161-5

48. Schneider MME, Hoepelman AIM, Schattenkerk JKME, et al. A controlled trial of aerosolized pentamidine or trimethoprimsulfamethoxazole as primary prophylaxis against Pneumocystis carinii pneumonia in patients with human immunodeficiency virus infection. N Engl J Med 1992; 327: 1836-41

49. Hardy WD, Feinberg J, Finkelstein DM, et al. A controlled trial of aerosolized pentamidine or trimethoprim-sulfamethoxazole as secondary prophylaxis against Pneumocystis carinii pneumonia in patients with human immunodeficiency virus infection. N Engl J Med 1992; 327: 1842-8

50. Simonds RJ, Hughes WT, Feinberg J, et al. Preventing Pneumocystis carinii pneumonia in persons infected with human immunodeficiency virus. Clin Infect Dis 1995; 21 Suppl. 1: S44-8

51. Luft BJ, Remington JS. Toxoplasmic encephalitis in AIDS. Clin Infect Dis 1992; 15: 211-22

52. Richards FO, Kovacs JA, Luft BJ. Preventing toxplasmic encephalitis in persons infected with human immunodeficiency virus. Clin Infect Dis 1995; 21 Suppl. 1: S49-56

53. Nightingale SD, Byrd LT, Southern PM, et al. Incidence of $M y$ cobacterium avium-intracellulare complex bacteremia in human immunodeficiency virus-positive patients. J Infect Dis $1992 ; 1082-5$

54. Nightingale SD, Cameron DW, Gordin FM, et al. Two controlled trials of rifabutin prophylaxis against Mycobacterium avium complex infection in AIDS. N Engl J Med 1993; 329: 828-33

55. Pierce M, Crampton S, Henry D, et al. A randomized trial of clarithromycin as prophylaxis against disseminated $\mathrm{Myco}$ bacterium avium complex infection in patients with advance acquired immunodeficiency syndrome. N Engl J Med 1996; 335: 384-91

56. Havlir DV, Dube MP, Sattler FR, et al. Prophylaxis against disseminated Mycobacterium avium complex with weekly azithromycin, daily rifabutin, or both. N Engl J Med 1996; 335: $392-8$

57. Ostroff SM, Spiegel RA, Feinberg J, et al. Preventing disseminated Mycobacterium avium complex disease in patients infected with human immunodeficiency virus. Clin Infect Dis 1995; 21 Suppl. 1: S72-6

58. Spector SA, McKinley GF, Lalezari JP, et al. Oral ganciclovir for the prevention of cytomegalovirus disease in persons with AIDS. N Engl J Med 1996; 34: 1491-7

59. Brosgart CL, Craig C, Hillman D, et al. A randomized, placebocontrolled trial of the safety and efficacy of oral ganciclovir for prophylaxis of CMV retinitis and gastrointestinal mucosal disease in HIV-infected individuals with severe immunosuppression [abstract no. LB-10]. Presented at the 35th Interscience Conference on Antimicrobial Agents and Chemotherapy; 1995 Sep 17-20; San Francisco. Washington, DC: The American Society for Microbiology, 1995

60. Rose DN, Sacks HS, Lan V. Cost-effectiveness analysis of oral ganciclovir to prevent cytomegalovirus disease in patients with AIDS. Presented at the 3rd Conference on Retroviruses and Opportunistic Infections; 1996 Jan 29-Feb 1; Washington, DC: The American Society for Microbiology, 1996

61. Powderly WG, Finkelstein DM, Feinberg J, et al. A randomized trial comparing fluconazole with clotrimazole troches for the prevention of fungals infection in patients with advanced 
human immunodeficiency syndrome. N Engl J Med 1995; 332: $700-5$

62. Polsky B, Gold JWM, Whimbey E, et al. Bacterial pneumonia in patients with the acquired immunodeficiency syndrome. Ann Intern Med 1985; 104: 38-41

63. Redd SC, Rutherford GW, Sande MA, et al. The role of human immunodeficiency virus infection in pneumococcal bacteremia in San Francisco residents. J Infect Dis 1990; 162: 1012-7

64. Simberkoff MS, El-Sadr W, Schiffman G, et al. Streptococcus pneumoniae infection in patients with acquired immune deficiency syndrome, with report of a pneumococcal vaccine failure. Am Rev Resp Dis 1984; 130: 1174-6

65. Huang KL, Ruben FL, Rinaldo CR, et al. Antibody responses after influenza and pneumococcal immunization in HIVinfected homosexual men. JAMA 1987; 257: 2047-50
66. Centers for Disease Control. Update on adult immunization: recommendations of the Immunization Practices Advisory Committee (ACIP). Pneumococcal disease. MMWR Morb Mortal Wkly Rep 1991; 40 (RR-12): 42-4

About the Author: Dr Simberkoff is the Chief, Infectious Diseases Section and Associate Chief of Staff for Research and Development, New York VA Medical Center and Associate Professor of Medicine. He has been involved in clinical trials since the beginning of the HIV epidemic.

Correspondence and reprints: Dr Michael S. Simberkoff, Chief, Infectious Diseases Section, New York VA Medical Center, 423 East 23rd Street, New York, NY 10010, USA. E-mail:SIMBERKOFF.MICHAEL_S+@NEW-YORK.VA.GOV 